\title{
DOI: 10.7596/taksad.v8i3.2250
}

Citation: Kuzmuk, O. (2019). Interconnectedness of the Philosophical and Aesthetical Views and Principles of Polyphonic Thinking in the Time of Baroque. Journal of History Culture and Art Research, 8(3), 293-305. doi:http://dx.doi.org/10.7596/taksad.v8i3.2250

\section{Interconnectedness of the Philosophical and Aesthetical Views and Principles of Polyphonic Thinking in the Time of Baroque}

\section{Olena Kuzmuk ${ }^{1}$}

\begin{abstract}
The article "Interconnectedness of The Philosophical and Aesthetical Views and Principles of Polyphonic Thinking in The Time of Baroque" is focused on the combinatorial method as the key compositional idea of polyphonic music of J. S. Bach; it emphasizes parallels between the scientific and artistic ideas of the time. The article also explores the interconnectedness of the philosophicalaesthetic, mathematical, and artistic cognition of the world in the Baroque period and its reflection in the music of J. S. Bach.

Polyphonic thinking is viewed as a creative phenomenon in the larger context of the historical development of Western European music. The article employs a complex method, a synthesis of various structural approaches ranging from the traditional methods of theoretical musicology to those of other sciences, structural linguistics in particular. Aria No. 3 from Bach's "Magnificat" and canon in four from his "Musical Offering" are brought into discussion as the works, which feature combinatorial method in Bach's instrumental and vocal-instrumental writing.

J. S. Bach uses combinatorial technique of two types - permutation and combination. Tones, motives, and larger thematic constructions could become subjects to permutations. The criterion for the selection of elements that participate in the combinatorial game is the principle of similarity and difference and the contrast between repetition and non-repetition, variability and immutability, which, in their turn, become manifestations of Ars combinatoria. The combinatorial method in Bach's music is not only its key compositional idea (at the level of operating with a limited number of intonational motifs-symbols in the infinitely large number of their combinations through different spatial relationships), but also the main factor in the continuous development of the dramatic idea, which corresponds to G. W. Leibniz's principle of "maximum and minimum."
\end{abstract}

Keywords: Polyphony, Combinatorics in music, J. S. Bach, G. W. Leibniz, Baroque, Ars combinatoria.

\footnotetext{
${ }^{1}$ Post-graduate student of National Musical Academy of Ukraine Named after P. I. Tchaikovsky, Kyiv, Ukraine. E-mail: olena.kuzmuk@gmail.com
} 


\section{Introduction}

Each historical period, epoch, and style is characterized by similarities between analytical and creative thinking. From the cultural point of view, art and science share a lot despite the fact that scientific thinking is primarily based on deterministic, logical modes of operation and veritable research data, while artistic - on innovative presentation of an artistic image in agreement with certain aesthetic goals and norms of the day. Scientific approach to numbers taken in their quality and interdependence is similar to artistic approach to word, sound, and color taken in their ability to form a dramatic composition. In the Baroque period, this similarity was manifested through a combinatorial method, which was linked to the art of polyphony. Combinatorics was and is one of the key logical-constructive principles of polyphony together with proportionality, mirror, and passacaglia principles.

Historical development of polyphony has been marked by certain common logical principles, typical for different historical periods, yet important for the time beyond those periods.

Let us name them.

i) principle of proportionality; its complex realizations are better illustrated by the polyphonic writing of the Gothic period (mensural rhythmics and multi-layered appearance of the isorhythmic motet, which refer to the invention of time-measuring mechanisms such as mechanical clock, which in the XIV century replaced sundial).

ii) combinatorial principle is one of the oldest historical principles of polyphony (one may already find vertical and horizontal movements of melodic formulas in the Stimmtausch (voice exchange) technique, in organum qaudruplum of Perotinus Magnus, and later in vertical, horizontal and twice convertible counterpoints of the codified Renaissance polyphony).

iii) mirror principle is also a historically old principle which means different things: imitation (direct symmetry), inversion (horizontal mirror symmetry), retrograde or cancrizans (vertical mirror symmetry), mensural mutations ('zooming in' and 'zooming out' of rhythmical length). Those 'things' appeared not earlier than in the art of Gothic and Renaissance periods and might have been inspired by optical inventions such as magnifying glass, telescope, and microscope.

iv) passacaglia principle, historically the latest one on the list and most representative of a newly born opera genre; despite its name it didn't originate from the genre of passacaglia and rather represented a certain approach to polyphony that allowed coexistence of ostinato, immutable thematic idea with mutability of musical material, most similar to a combination of static image and its dynamic development in the opera.

\section{Methods}

This article employs a synthetic approach to the structural analysis of the musical text. It uses a complex method, a combination of various structural approaches ranging from the traditional methods of theoretical musicology to those of other sciences, structural linguistics in particular. It also represents a synthesis of traditional musicology and methods of cultural analysis used by French scholar Abraham Moles (Moles, 1975). Moles perceived culture as a metalanguage with its own 
"dictionary" and key components of thinking which can be written down with a certain "alphabet," which, in its turn, can be used for culture's content analysis.

Thus, by defining key systemic elements of different levels one may trace their interaction and order of appearance (in time and space). This essentially structuralist approach can be applied to the music of different periods, J. S. Bach's music in particular.

While doing structural analysis of J. S. Bach's polyphonic technique, augmented by traditional analytical approaches, one should pay attention to similarities and dissimilarities, repetition and nonrepeatability and identify elements that can form certain spatial configuration, combine into permutational blocks, and integrated hierarchical levels of musical structure. The reasons for the particular attention to each element are its discreteness, clear separation from the general musical fabric, especially when the relationships between relief and background, repeatability and contrast are considered.

Such elements in the music of J. S. Bach can be motives, phrases, and larger constructions. An important component of the analysis of the combinatorial method in J. S. Bach's music is the repeatability of the elements (melodic-rhythmic figures and thematic constructions) and their positioning in regards to other elements.

Perception of the world as an alphabet or book (typical of the Baroque period) resonates well with a structural approach, and helps us to better understand combinatorial thinking in J. S. Bach's music.

\section{Background}

A. V. Lebedeva's dissertation thesis "Ars Combinatoria and Musical Practice of the 18th Century" (Lebedeva, 2002) was dedicated to the study of combinatorics in the Baroque period. L. L. Gerver paid special attention to polyphonic thinking and the combinatorial method in the works of the Early Baroque and Classical composers Girolamo Frescobaldi and W. A. Mozart. (Gerver, 1996; Gerver, 2006). M. R. Chernaya discussed combinatorics in the texture and syntax of W. A. Mozart's works and in his compositional technique (Chernaya, 2013).

Among the English-language studies, the most significant one was L. G. Ratner's essay "Ars combinatoria: Chance and Choice in 18th century Music" (Ratner, 1970).

Despite the large number of studies of musical combinatorics in the Baroque era, we still lack works on the combinatorial method in the music of J. S. Bach, with the sole exception of an article by I. B. Pyaskovsky “Bach and Leibniz: Resonances of Their Worldviews" (Piaskovsky, 2008).

Hence this researcher's attention to the combinatorial method as the central compositional idea of polyphonic music of J. S. Bach, which further emphasizes parallels between the scientific and artistic ideas of the time. It is this attention that reveals truly original approach and scholarly novelty, complementing and expanding the contemporary view of Bach's polyphonic mastery.

Combinatorics as a compositional method was widely used in the new (Charles Ives, Pierre Boulez, Yannis Xenakis, Milton Babbitt, Krzysztof Penderecki) and contemporary (Sergei Slonimsky, 
Valentin Silvestrov, Leonid Hrabovsky, Alex Voytenko, Andrey Merhkel) music. This is also why a detailed study of the combinatorial method in the music of J. S. Bach has much relevance to us today.

There have been many studies which analyzed the interaction of scientific and artistic thinking. For example, G. Vasyutochkin's analysis of Scott Buchanan's “Poetry and Mathematics (1962) deals with analogies drawn by Buchanan between numerical relations and verbal texts presented as certain mathematical ratio between the development of literary plot (which requires limited number of characters and subplots) and possible combinations of elements in a pre-established system that rules logical game. According to Vasyutochkin, "author's take on plot's structure is similar to the ideas of (Russian) Formalists, who viewed plot as a combination of certain elements. Buchanan's analogy between different versions of the plot and the chess-playing also brings us back to the ideas of the Formalist School. Chessboard figures operate within firmly defined rules and those figures' validity largely remains the same" (Vasyutochkin, 1968, p. 381-382).

\section{Results}

The purpose of this article is to expose the interconnectedness of the philosophical-aesthetic, mathematical, and artistic cognition of the world in the Baroque period and its reflection in the music of J. S. Bach. Using philosophical ideas of G. W. Leibniz from his treatise "Ars combinatoria," I will discuss the specifics of artistic application of the combinatorial method in the polyphonic music by J. S. Bach.

The close relationship between scientific and artistic cognition of the world was typical of the Baroque era. At that time, artistic forms and genres, poetic language and rhetoric, the style of research treatises, methods of representing scientific concepts mutually influenced each other.

Often the same text contained both scientific and artistic information. Scientists included poetic fragments into their treatises and used highly poetic language to describe their ideas. This was a manifestation of a desire, so typical of the Baroque period, to create a universal language for the description of the world. The proximity of the scientific and artistic forms of representation of knowledge has already been noted by a Russian scholar A. V. Mikhailov: "The poets of the Baroque era create not just lyrical or narrative poems, novels $<\ldots .>$ they, in essence, create everything that one way or another may be included in the compendium - the one that is able to represent the world in its entirety $\langle\ldots\rangle$ Thus, the work of art can be based either on a well-known numerical calculation, or on some substantive principle, which cannot be possibly grasped by the reader, yet, in some instances, may be accessed by scientific analysis." (Mikhailov, 1997, p. 120-121).

The consistency of musical utterance and the introduction of refined order into its structure are in full agreement with the rationalistic attitudes of the era. The desire for seeing formal logic ruling supreme led to the penetration of combinatorial principles into many chapters of the theory of composition. Chords, rhythmic-melodic figures, tonal plans, and elements of musical form all became subjects to permutations.

L. G. Ratner (Ratner, 1970) identifies four types of application of formal logic to the theory of composition: 
1) generation and combination of musical motifs and phrases

2) melodic and harmonic permutations within the fixed structure of the musical phrase

3) permutations in the order of tonalities (tonal plans)

4) permutations of parts of musical form

Thus, he deals with different levels of musical structure and sees combinatorics as a method of creating and organizing musical material in the genres relevant to the historical period.

A theorist of musical combinatorics Joseph Riepel speaks of the permutations of tones in the composition of figures, then - the figures and motives in the composition of the phrases or periods; and also the possibility of building up a melody with different versions of a tonal plan.

The idea of combinatorics, as it has been formulated by G. W. Leibniz, correlates with the principle of invention, inventio, one of the most important artistic and aesthetic principles of the Baroque period. Combinatorics is a response to Zeitgeist which has already been expressed in the game thinking, the popularity of many combinatorial games, various theories of "sophisticated mind", and in pursuit of wit as an "art of quick reason."

The aesthetics of wit was reflected in the treatises "Wit, or the Art of Quick Mind" by B. Gracian, "Trattato delle Acutezze" by M. Pellegrini.

According to Leibniz, the combinatorial component is evident in any creative act. The very term "combinatorics" was first used by G. W. Leibniz in his work "Reflections on Combinatorial Art" ("Ars combinatoria", 1666). And the doctrine of Ars combinatoria was reflected in many theoretical treatises of that time (authored by Marin Mersenne, Athanasius Kircher, Joseph Riepel, G. K. Koch); it had a significant impact on musical aesthetics and use of musical material in the periods of Baroque and Classicism.

M. N. Lobanova in her book on aesthetics and poetics of Baroque period in Western Europe notes that "Ars combinatoria" in music is a sort of "universal language" (Lobanova, 1994, p. 176). According to Lobanova, the artistic principles, which govern music, parallel the universal character of logic described by Leibniz with the help of mathematical symbols. Joseph Riepel in his treatise "Basic Rules of the Sound Law in General" (1755) stated that musical and linguistic constructions were equivalent to each other.

J. P. Kirnberger also emphasized the basic identity of musical and spoken language structures: "Chords in music are the same as words in a language: a sentence is formed in our speech from several connected and meaningful expressive words, and musical sentence or period is born from several interconnected chords, which end in cadenza. Like spoken sentences connected to each other create the flow of speech, so do many interconnected musical periods result in a piece of music "(quoted from Zakharova, 1981, p. 115).

The combinatorial method in music was first developed by Raymond Llull in his treatise "Great Art" (Ars Magna), and by Llull's follower Athanasius Kircher. In Ars Magna, Llull argued that any discovery was possible on the basis of a combination of a limited number of elements. Kircher's work "Musurgia Universalis" (1650) contained a description of a musical turntable, a "wonderful music arithmetic" (musarithmica mirifica), which can be used in combinatorial composition of melodies. 
And G. W. Leibniz provided us with the following definition of combinatorics:

"Combinatorial art <...> is such a science (which can be, generally speaking, called characteristic or specific one) that deals with the forms of things or formulas of the universe, i. e., with quality in general, or with similar or dissimilar, as those or other formulas originate from combinations of $a, b, c$, etc. Therefore, algebra obeys combinatorics and constantly uses its rules, which, however, are more general and are relevant not only in algebra, but also in the art of interpretation, in various types of games, in geometry itself <...> finally, everywhere where similarity is present." (Gerver, 1996, p. 122).

This definition testifies to Leibniz's understanding of combinatorics as a universal science dealing not only with the form, quality, quantity of objects and phenomena but also with their essence. In spite of the fact that combinatorial art operates with numbers, it is inappropriate to confine it to a purely mathematical science; for a number (as one of the elements of the combinatorial method) is a code, which refers to both tangible things and generalized ideas.

In the following definition of combinatorics, while speaking of the instances when quantity of dissimilar things brought together results in some new quality, the philosopher emphasizes the dialectical nature of the art in question: " $\mathrm{A}() \mathrm{fter}$ all, the main feature of combinatorial art, is to produce, from a comparison of an entirely different material, some new useful things" (Gerver, 1996, p. 429).

The universality of this art is further confirmed in G. W. Leibniz's appeal to the language of signs and his understanding of a sign (numerical, alphabetic, musical, etc.) as the key operational element of the combinatorial method: "With the help of the signs denoting both (definite) numbers and indefinite numbers of letters of the alphabet used in various combinations, one may miraculously express bodily figures. This is commonly called symbolic calculus by means of characteristic signs, or images of things <...> Although the numerical science has reached a very high degree of perfection and with the art of combinatorics, or general symbolism (speciosa generelis), applied to the numbers the mathematical analysis has been born, this science may achieve even more" (Gerver, 1996, p. 447).

The latter description confirms that the art of combinatorics goes far beyond the specifically scientific subjects. According to the philosopher, combinatorics is a metascience, which operates with generalized ideas and symbols: "T()hanks to some kind of combinatorial art, one can advance so much in metaphysics, ethics, and any other area of reasoning that are not related to figurative representations themselves, that those areas become accessible to imagination, like algebraic numbers, due to the notation with characteristic signs and letters of the alphabet (Gerver, 1996, p. 449)."

Summing up G. W. Leibniz's thoughts on the combinatorial art, we can draw the following conclusions.

1. Combinatorics is the art of synthesis of all possible sciences and teachings and a possibility of achieving a qualitatively new result by combining many different ideas in a single unifying aspect.

2. Combinatorics is the art of invention, when a combination of familiar images results in a qualitatively new image, not associated with any of the initial images brought together. 
3. Combinatorics is the art of symbolic manipulation of images using characteristic signs (letters of the alphabet, numbers, significations).

4. Combinatorics is the art of comparison and a relationship based on similarity and dissimilarity.

5. Combinatorics is the art of rearranging the minimum number of elements, which gives rise to the maximum number of possible combinations of these elements. (Here one can see practical application of Leibniz's principle of "maximum and minimum": "The formulation of this principle was determined by the interpretation of the world sequence of monads, according to which the "beginning" of the series is rooted in a sequence of infinitely small, and the "end" leads to an infinite increase. The principle itself may be formulated as follows: the minimum of the existing generates the maximum of existence" (Leibniz, 1984, p. 86)).

The idea of maximum and minimum was reflected in the treatise of the German musical theorist C. G. Schröter ("Deutliche Anweisung zum Generalbass", 1772), who derived all chords, including the chords not containing any thirds, from one major triad by various modifications (such as interchange, transposition, conversion; octave replacement by septima; introduction of non-chord sounds).

Scholar M. R. Chernaya in her study of the combinatorics in the music of W. A. Mozart, has noted that the mathematical term "combinatorics" retained its meaning in regard to music as "a set of methods of systemic organization and arrangement of a certain number of objects in agreement with the given rules." (Chernaya, 2013, p. 131). Those rules are combination, permutation, and placement.

Two types of combinatorics are mentioned in the treatises of the Baroque period: permutation (rearrangement of objects according to the pattern $A B C D A C B D C A B D$, etc.) and combination (replacement of one or more objects with the similar purpose - ABCD ABED AECD)2.

Consider the features of the combinatorial method in the instrumental and vocal-instrumental music of Bach. For example, in Aria No. 3 of the "Magnificat," the main, cross-cutting theme consists of five elements.

The element of the musical composition, which becomes the object of the combinatorial game, is the thematic motive.

The very presentation of the subject indicates variative combinations of the presented intonation motifs, because all of them, in a thematic sense, are equal and derived from one intonational idea. The subsequent presentation of theme in the vocal and instrumental parts is characterized by constant variations in its intonational, motivic (as the result of rearrangement) and tonal aspects.

Example 1

2 The term "combination" has been introduced by B. Pascal (1665), and the term "permutation" - by J. Bernoulli. 

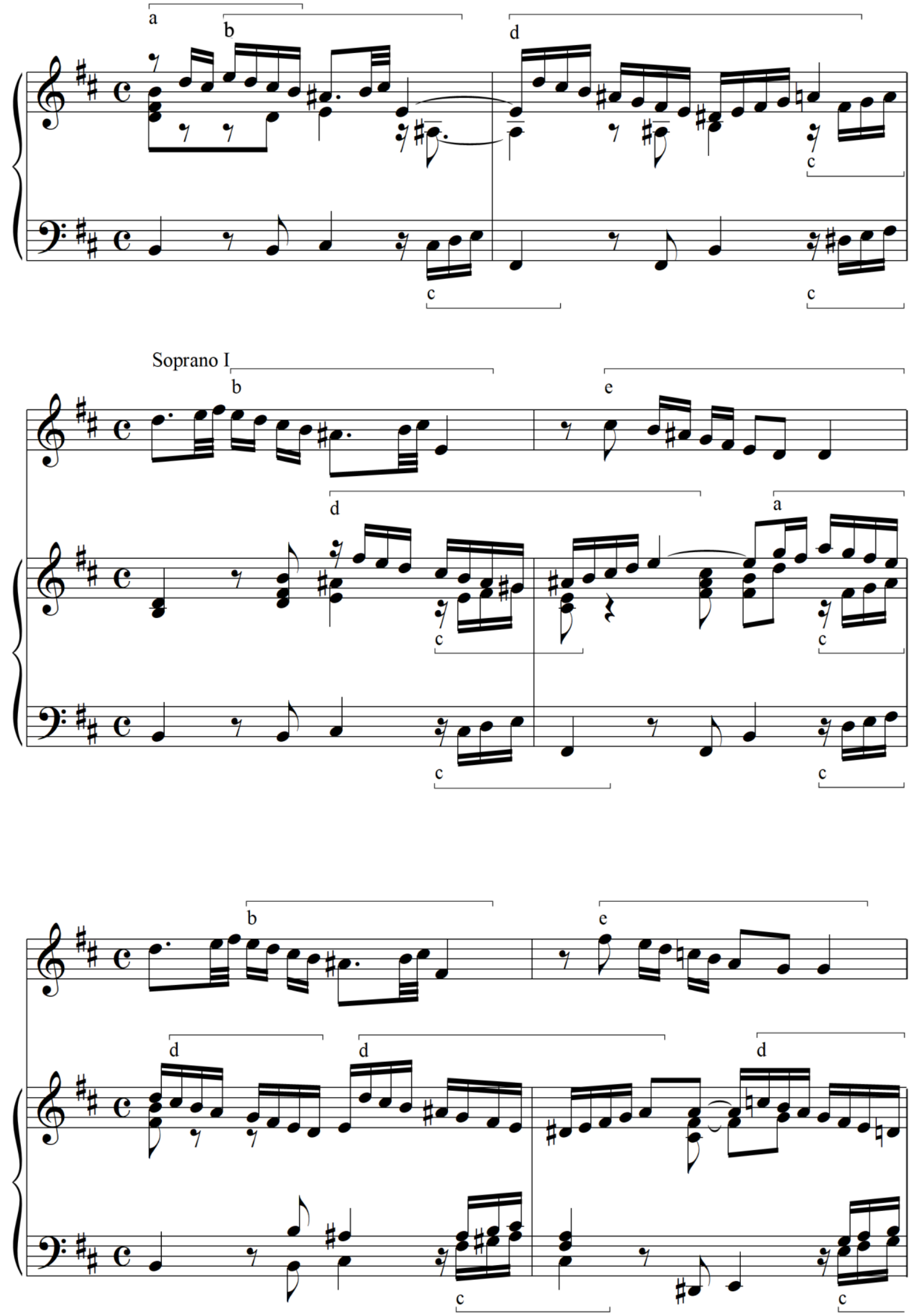
Scheme 2 reveals combinatorics of cross-cutting motifs in the initial presentation of subject and its two subsequent presentations:

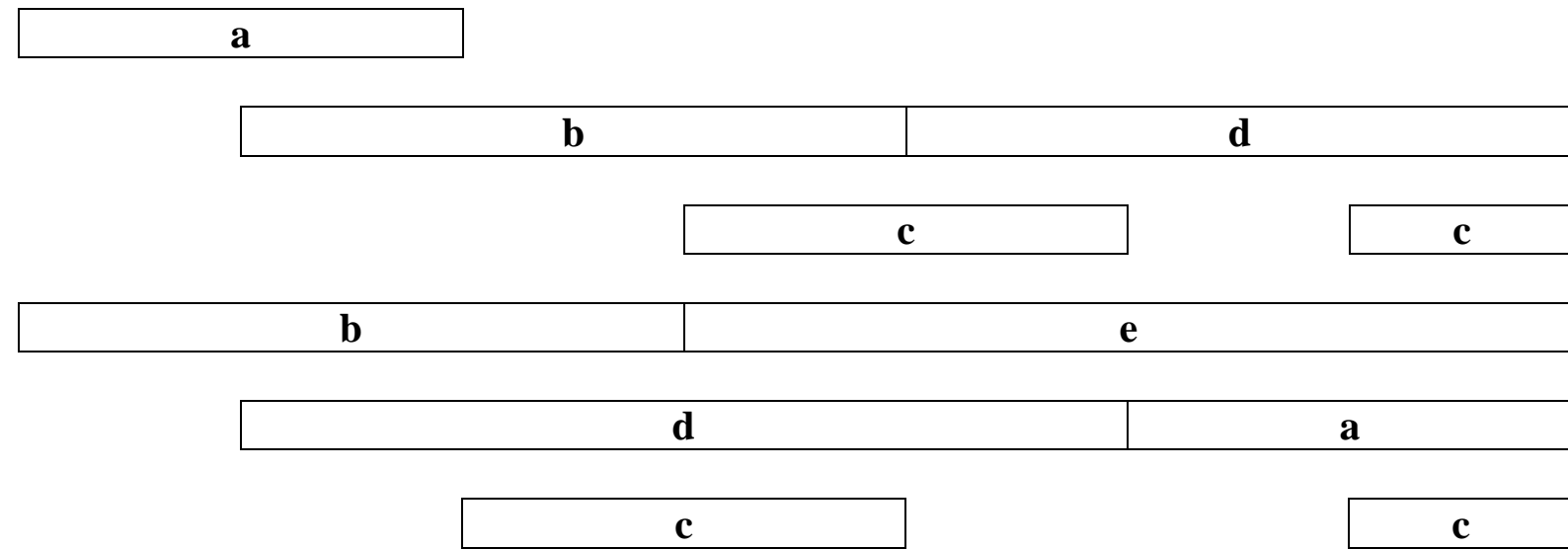

\begin{tabular}{|c|c|c|}
\hline \multicolumn{1}{|c|}{ b } & \multicolumn{2}{c|}{ e } \\
\hline d & d & d \\
\hline & c & c \\
\hline
\end{tabular}

Combinatorial permutation is realized both vertically and horizontally, i. e. in different spatial directions, by means of convertible counterpoints.

Thus, combinatorial method in Aria's composition is realized through predetermined permutations of thematic elements moving both vertically and horizontally and leads to perpetual variations in subject's intonation and structure, to unstoppable dramatic development and, ultimately, to renewal of musical material. The renewal is further supported by a type of combination when one element $(C)$ is replaced by a similar one $(E)$, while the third one $(A)$ is eventually lost $(A B C D-B D E A-$ DBDE).

Let us take canon in four from "The Musical Offering" of J. S. Bach as another clear example of a highly regimented combinatorial permutation of thematic elements in both vertical and horizontal directions. In his article "Bach and Leibniz: Parallels in Their Worldviews" (Piaskovsky, 2008, p. 141) I. B. Piaskovsky provides a scheme for this endless canon and draws attention to a very high number of variations (the total of 24) of the way each voice is introduced and links it to the principle of "maximum and minimum," also evident in a notation for one voice which stands or the total of $1 / 8$ of time this canon is played.

This endless type of canon has been previously used in the $14-15^{\text {th }}$ century English rondellus. Bach employs a preexisting form and enriches it with bright thematic material. Not the entire motifs but the thematic elements $A, B, C, D$, quite distinct in their intonation, become the subjects of permutations. 
Example 2.

A
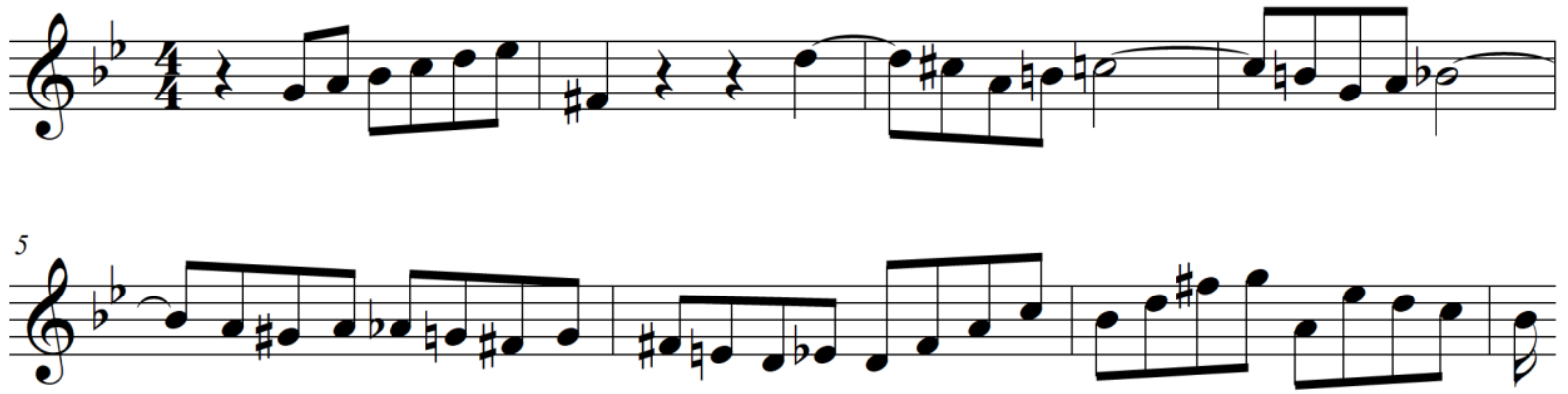

B
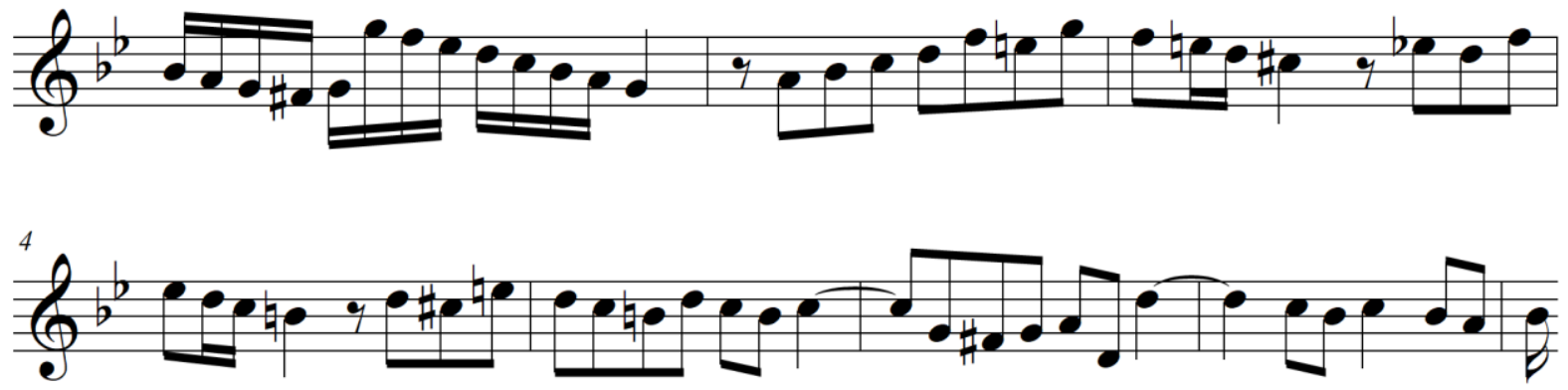

C
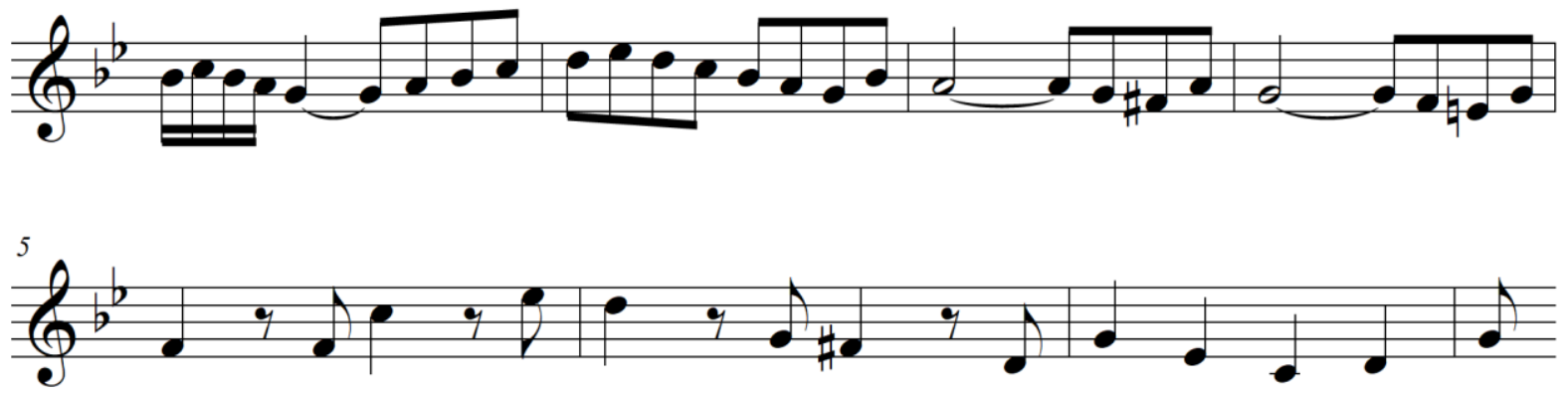

D
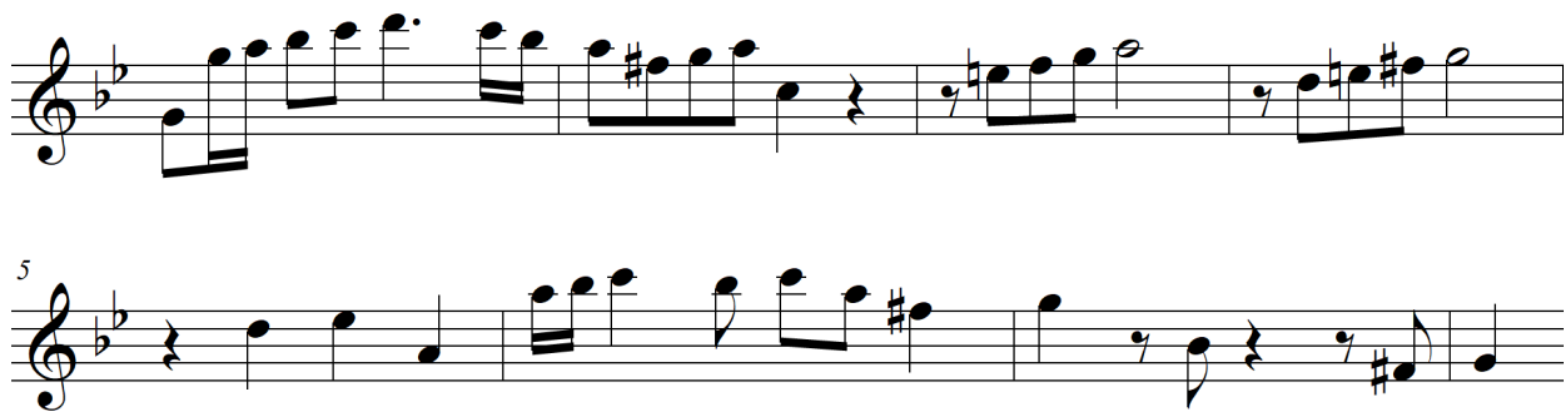

If we see not only section $A$, but also sections $B, C$, and $D$ as the material for proposta, our attention will move, in each case, to other thematic blocks. As a result, four voices would sound 
identically; as for us, listeners, we would start paying more attention to the thematic material of proposta.

Example 3
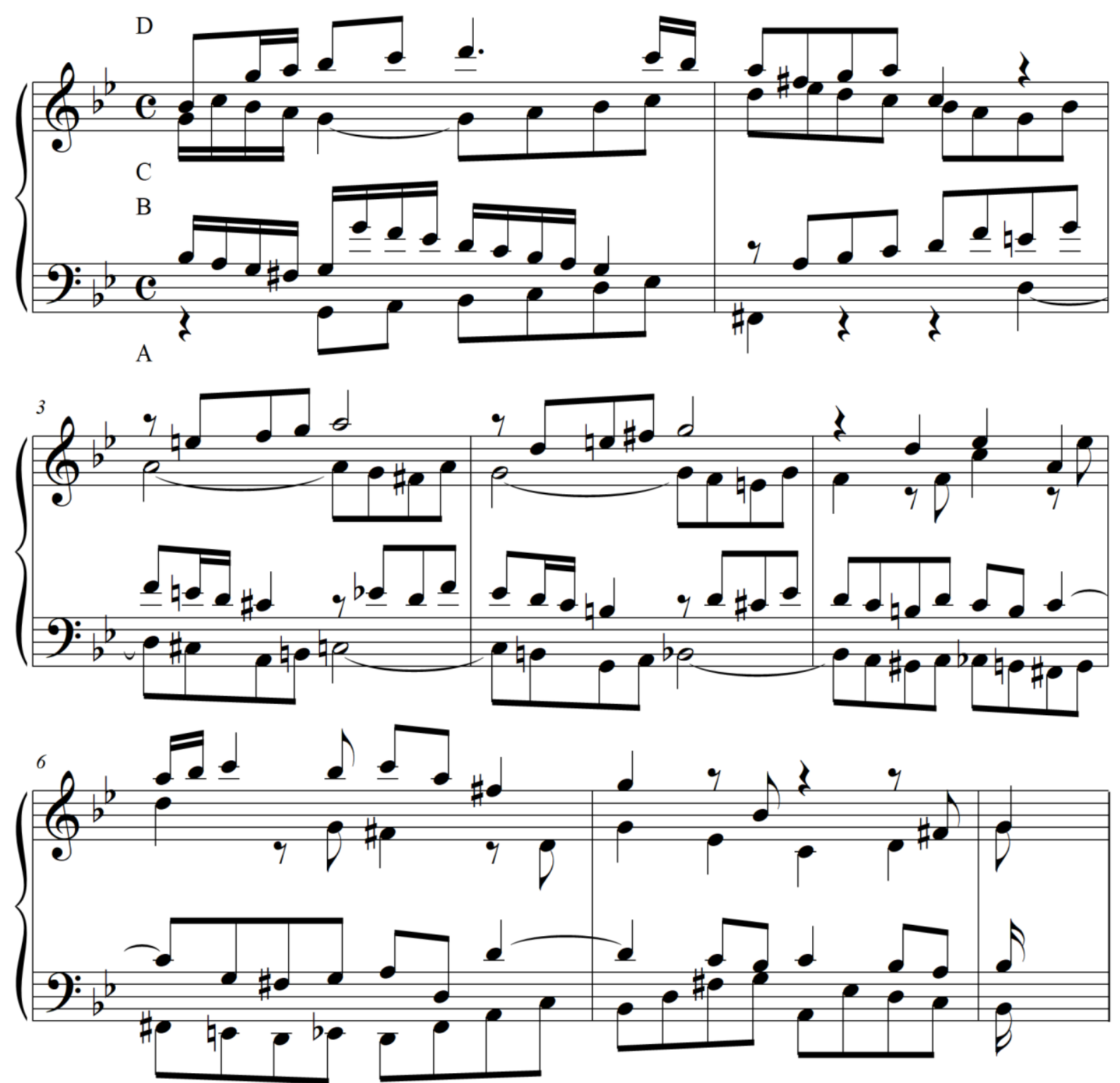

Scheme for J. S. Bach's canon in four

\begin{tabular}{|c|c|c|c|c|c|c|c|c|c|c|}
\hline $\mathbf{A}$ & $\mathbf{B}$ & $\mathbf{C}$ & $\mathbf{D}$ & $\mathbf{A}$ & $\mathbf{B}$ & $\mathbf{C}$ & $\mathbf{D}$ & & & \\
\hline & $\mathbf{A}$ & $\mathbf{B}$ & $\mathbf{C}$ & $\mathbf{D}$ & $\mathbf{A}$ & $\mathbf{B}$ & $\mathbf{C}$ & $\mathbf{D}$ & & \\
\hline & & $\mathbf{A}$ & $\mathbf{B}$ & $\mathbf{C}$ & $\mathbf{D}$ & $\mathbf{A}$ & $\mathbf{B}$ & $\mathbf{C}$ & $\mathbf{D}$ & \\
\hline & & & $\mathbf{A}$ & $\mathbf{B}$ & $\mathbf{C}$ & $\mathbf{D}$ & $\mathbf{A}$ & $\mathbf{B}$ & $\mathbf{C}$ & $\mathbf{D}$ \\
\hline
\end{tabular}

\begin{tabular}{|c|c|c|c|c|c|c|c|c|c|c|}
\hline $\mathbf{B}$ & $\mathbf{C}$ & $\mathbf{D}$ & $\mathbf{A}$ & $\mathbf{B}$ & $\mathbf{C}$ & $\mathbf{D}$ & $\mathbf{A}$ & & & \\
\hline & $\mathbf{B}$ & $\mathbf{C}$ & $\mathbf{D}$ & $\mathbf{A}$ & $\mathbf{B}$ & $\mathbf{C}$ & $\mathbf{D}$ & $\mathbf{A}$ & & \\
\hline & & $\mathbf{B}$ & $\mathbf{C}$ & $\mathbf{D}$ & $\mathbf{A}$ & $\mathbf{B}$ & $\mathbf{C}$ & $\mathbf{D}$ & $\mathbf{A}$ & \\
\hline & & & $\mathbf{B}$ & $\mathbf{C}$ & $\mathbf{D}$ & $\mathbf{A}$ & $\mathbf{B}$ & $\mathbf{C}$ & $\mathbf{D}$ & $\mathbf{A}$ \\
\hline
\end{tabular}




\begin{tabular}{|l|l|l|l|l|l|l|l|l|l|l|}
\hline $\mathbf{C}$ & $\mathbf{D}$ & $\mathbf{A}$ & $\mathbf{B}$ & $\mathbf{C}$ & $\mathbf{D}$ & $\mathbf{A}$ & $\mathbf{B}$ & & & \\
\hline & $\mathbf{C}$ & $\mathbf{D}$ & $\mathbf{A}$ & $\mathbf{B}$ & $\mathbf{C}$ & $\mathbf{D}$ & $\mathbf{A}$ & $\mathbf{B}$ & & \\
\hline & & $\mathbf{C}$ & $\mathbf{D}$ & $\mathbf{A}$ & $\mathbf{B}$ & $\mathbf{C}$ & $\mathbf{D}$ & $\mathbf{A}$ & $\mathbf{B}$ & \\
\hline & & & $\mathbf{C}$ & $\mathbf{D}$ & $\mathbf{A}$ & $\mathbf{B}$ & $\mathbf{C}$ & $\mathbf{D}$ & $\mathbf{A}$ & $\mathbf{B}$ \\
\hline
\end{tabular}

\begin{tabular}{|c|c|c|c|c|c|c|c|c|c|c|}
\hline $\mathbf{D}$ & $\mathbf{A}$ & $\mathbf{B}$ & $\mathbf{C}$ & $\mathbf{D}$ & $\mathbf{A}$ & $\mathbf{B}$ & $\mathbf{C}$ & & & \\
\hline & $\mathbf{D}$ & $\mathbf{A}$ & $\mathbf{B}$ & $\mathbf{C}$ & $\mathbf{D}$ & $\mathbf{A}$ & $\mathbf{B}$ & $\mathbf{C}$ & & \\
\hline & & $\mathbf{D}$ & $\mathbf{A}$ & $\mathbf{B}$ & $\mathbf{C}$ & $\mathbf{D}$ & $\mathbf{A}$ & $\mathbf{B}$ & $\mathbf{C}$ & \\
\hline & & & $\mathbf{D}$ & $\mathbf{A}$ & $\mathbf{B}$ & $\mathbf{C}$ & $\mathbf{D}$ & $\mathbf{A}$ & $\mathbf{B}$ & $\mathbf{C}$ \\
\hline
\end{tabular}

Therefore, the combinatorics is applied both vertically and horizontally. Let us discuss, for example, a fragment for four voices from bar $24 \mathrm{on}$. It is obvious that the voice, which has started in proposta, presents the following combination of thematic elements: DABC. The voice of the first risposta provides us with $C D B A$, the voice of the second risposta is based on $B C D A$, while the fourth voice (of the third risposta) presents the following sequence: $A B C D$. At the same time, vertical thematic relations are also subjects to the combinatorics. By drawing vertical lines from the third risposta to proposta one may see the following sequences: $A B C D, B C D A, C D A B$ and $D A B C$. In other words, all horizontal relations are repeated vertically.

Here we deal with permutation combinatorics where larger thematic combinations become the subject of further permutation. Permutation technique "creates a multitude of dissimilar new forms from a limited number of initial elements" (Moles, 1975, p. 100 - 101).

\section{Conclusion}

As we see, J. S. Bach uses combinatorial technique of two types - permutation and combination. Tones, motives, and larger thematic constructions could become subjects to permutations. The criterion for the selection of elements that participate in the combinatorial game is the principle of similarity and difference and the contrast between repetition and non-repetition, variability and immutability, which, in their turn, become manifestations of Ars combinatoria.

Bach creates the universe of sounds, guided by rational laws and mathematical methods. The rationality and mathematical nature of his compositional thinking allow us to draw an analogy between the artistic and scientific worldviews of the Baroque era. Finding structural elements that become subjects of combinatorial permutations allows us to understand the patterns of J. S. Bach's logic and musical thinking and opens a possibility for producing a musical text in his style.

Thus, the combinatorial method in Bach's music is not only its key compositional idea (at the level of operating with a limited number of intonational motifs-symbols in the infinitely large number of their combinations through different spatial relationships), but also the main factor in the 
continuous development of the dramatic idea, which corresponds to G. W. Leibniz's principle of "maximum and minimum."

\section{References}

Chernaya, M. R. (2013). Combinatorial Paradigm in the Composition of Clavier Sonatas by W. A. Mozart.Vestnik KemGUKI. No. 25. Kemerovo.

Gerver, L. L. (1996). Ars Combinatoria in the Music of Mozart. In: Mozart: Problems of His Style. Moscow: RAM.

Gerver, L. L. (2006) Ars Combinatoria and Polyphonic Technique of G. Frescobaldi. In: From Guido to Cage: Studies in Polyphony. Moscow: TS-PRIMA.

Lebedeva, A. V. (2002). Ars Combinatoria and Musical Practice of the $18^{\text {th }}$ Century. Dissertation thesis. Moscow.

Leibniz, G. W. (1984). Works in 4 volumes, Vol. 3. Moscow: Mysl',

Lobanova, M. N. (1994). Western European Musical Baroque: Aesthetics and Poetics. Moscow: Muzyka.

Mikhailov, A. V. (1997). Baroque Poetics: The End of Rhetoric Period. In: Languages of Culture, ed. N. S. Pavlov, S. Yu. Khurumov. Moscow: Yazyki russkoy kul'tury.

Moles. A. (1975). Art and Computer. In: A. Moles, W. Fuchs, M. Kassler. Moscow: Mir.

Moles. A. (1973). Sociodynamics of Culture. Moscow.

Narsky, I. (1972). Gottfried Leibniz. Moscow: Mysl'.

Piaskovsky, I. B. (2008). Bach and Leibniz: Resonances of Their Worldviews. In: Musical Creativity and Research in Historical Perspective. Vol. 73. Kiev.

Ratner, L. G. (1970). Ars Combinatoria. Chance and Choice in the 18 century Music. In: Studies in the 18 century Music. London, 1970.

Vasyutochkin, G. (1968). On Artistic and Mathematical Thinking. In: Friendship of Sciences and Secrets of Creativity. Moscow: Iskusstvo.

Zakharova, O. I. (1981). Rhetoric and German Music of the $17^{\text {th }}-$ First Half of the $18^{\text {th }}$ Century. Dissertation thesis. Moscow. 